\title{
Vibroacoustic response of stiffened thin plates to incident
}

\author{
sound \\ Dong Zhao ${ }^{1^{*}}$, Giacomo Squicciarini ${ }^{1}$ and Neil Ferguson ${ }^{1}$ \\ ${ }^{1}$ Institute of Sound and Vibration Research, University of Southampton, Southampton SO17 1BJ, UK
}

\section{Abstract}

A model was developed and applied for predicting the vibroacoustic response of stiffened plates excited by different incident sound fields. A relationship between the Joint Acceptance Function (JAF) and the modal radiation efficiency is derived. This allows a direct calculation for both the vibration response and the sound transmission loss $(\mathrm{TL})$ in the presence of a diffuse field whilst accounting for full vibroacoustic coupling. The response for different structural configurations, plane versus diffuse acoustic excitation and modelling approximations are highlighted, so presenting a systematic quantification of the factors influencing the vibroacoustic response. It is found that vibroacoustic coupling is enhanced by the presence of stiffeners, with the effects for different types of acoustic excitation and the contribution from the modal cross terms being most significant for the TL results. A coupled FE-BE numerical model of stiffened plate and other empirical formulae for unstiffened plate are subsequently used for the validation and verification of the proposed model. The results show that the computational cost of simulation can be significantly reduced with a small loss of accuracy when using the proposed model in comparison to the fully coupled FE-BE numerical model. Keywords: Vibroacoustic coupling, Joint Acceptance Function, sound transmission loss

\footnotetext{
* Corresponding author:

Dong Zhao, Institute of Sound and Vibration Research, University of Southampton, Southampton SO17 1BJ, UK Email: dz1r18@soton.ac.uk
} 


\section{Introduction}

Structures characterised by high-stiffness and low-mass are typically required in aerospace sectors; examples include launch vehicles fairings and aircraft fuselage. Stiffened, thin isotropic plates are often used in this context. These structures can be excited by high intensity broadband sound fields and the vibroacoustic response can subsequently lead to major concerns for acoustic fatigue and sound transmission. In addition, thin plates can exhibit important vibroacoustic coupling phenomena that on some occasions need to be taken into account.

For a general structure with complex geometry, a possible approach to study its interaction with an incident sound field is to develop a coupled FE-BE model [1-4]. In this case, the structural and acoustic domains, modelled by a structural FE and acoustic BE model, are coupled at their common surface thus taking into account compatibility and equilibrium conditions. Whilst this approach offers a great level of accuracy at low-frequency, it suffers from a very heavy computational cost due to the fine mesh required as the frequency increases [5]. The cost can even be prohibitively high for Monte Carlo simulations, which are required to numerically produce an ensemble of diffuse fields [6]. Generally, because of the low density of air compared to the structural material, the re-radiated pressure field is small enough to have a negligible effect on the structural vibration. Consequently, an uncoupled solution can also be adopted; the structural and acoustic domains would then be modelled independently. The vibration response, or the transmitted sound power, can be calculated by using an equivalent force excitation [7]. However, the uncoupled solution may lead to an inaccurate result for a thin structure, especially in the case of a structure undergoing a large deflection $[8,9]$ and the computational cost in a single BEM model is still demanding due to the fully populated matrices.

Alternatively, the response of plate-like structures can be calculated by making use of the Joint Acceptance Function (JAF). This is defined as the ratio between the modal force due to a specific sound field and the overall force due to a uniform pressure field [10]. The JAF for a harmonic acoustic sound field acting on a rectangular plate can be written as [11]

$$
j_{n}(\omega)=\frac{\int_{0}^{b} \int_{0}^{a} \psi_{n}(x, y) p(x, y, \omega) \mathrm{d} x \mathrm{~d} y}{p_{0} a b}
$$

where $\psi_{n}(x, y)$ is the mode shape of the $n^{\text {th }}$ mode, $\omega$ is the angular frequency, $a$ and $b$ are the dimensions of the plate and $p_{0}$ is an equivalent uniform pressure field. In the case of baffled plates, this corresponds to a doubling of the incident pressure field amplitude ignoring the re-radiation. The JAF indicates how efficiently the $n^{\text {th }}$ mode can be excited when subjected to a particular pressure field in comparison to a uniform excitation. 
The sound radiation can be represented in terms of modal radiation efficiencies, which represent how efficiently the vibration of one mode can radiate sound power in comparison to a vibrating piston with the same mean square average velocity. The radiation efficiencies are typically used to predict the sound radiation due to external forces, but they can also represent vibroacoustic coupling in terms of modal radiation damping. The modal radiation damping $\sigma_{n}$ is defined as [12]

$$
\sigma_{n}(\omega)=\frac{W_{n}}{\rho_{0} c a b\left\langle\overline{v_{n}^{2}}\right\rangle}
$$

where $W_{n}$ is the acoustic power radiated by $n^{\text {th }}$ mode and $\left\langle\overline{v_{n}^{2}}\right\rangle$ is the spatial-averaged mean-square normal velocity of the plate for $n^{\text {th }}$ mode.

In the literature, the JAF has been used to evaluate the response of isotropic plates in the presence of various types of sound fields such as a progressive plane wave, diffuse field and turbulent boundary layer (TBL). One of the earliest work in this area was presented by Wallace in [13]. The mean square displacement of a simply supported isotropic plate subjected to a diffuse field was predicted by averaging the Joint Acceptance Function (JAF) over all spatial incident field directions in the half-space above the plate. Blevins [14] developed an approximate method, assuming that the pressure field and the mode shape have the same spatial distribution, which effectively makes the JAF equal to unity. Coyette [15] extended the JAF to include a structure comprising a truncated conical geometry and provided an efficient method to evaluate the JAF for a diffuse field. However, these models neglected the full vibroacoustic coupling and cross modal contributions.

Fahy [4] presented an approach that included vibroacoustic coupling as well as cross modal contributions for an infinite strip subjected to an incident plane wave. The reflected acoustic field was essentially decomposed into two parts: the sound produced by reflection or scattering from an infinitely rigid surface, producing a blocked force on the structure, and radiated sound due to a forcing term corresponding to the re-radiated pressure field. Compared to a non-coupled solution, the effect of vibroacoustic coupling resulted mostly in added damping for thin plates in air. Mejdi [16] extended this analysis to stiffened plates by using a modal expansion technique together with the full radiation impedance matrix. The spatial-averaged mean square velocity and radiated power due to a point source excitation were predicted and compared with the FEM and BEM formulation to examine the effect of cross modal contributions. The response to a diffuse field was also evaluated by using the field incidence approximation [7] and compared with measurements.

This paper proposes a modal model that predicts the vibration response and sound transmission through stiffened panels excited by different forms of incident sound field. Vibroacoustic coupling is 
maintained in the solution by deriving relationships between the modal radiation efficiency and the JAF for baffled stiffened plates. The proposed approach provides a new framework for evaluating the coupled vibroacoustic response of flat plates, stiffened or not, to incident sound in terms of some of the existing formulated vibroacoustic quantities (radiation efficiency and/or Joint Acceptance Functions). Compared to similar studies ([4] [16]) , the proposed approach gives a concise step by step formulation which only requires the in-vacuo modal parameters and extends the applicability to finite, stiffened plates with arbitrary boundary conditions subjected to either plane wave or diffuse field excitation. In addition, a significant computational reduction is achieved whilst retaining good accuracy compared to a fully coupled, numerical FE-BE solution. The model is described in Section 2 and example results are presented in Sections 3 and 4 for the structural model, dynamic response and sound transmission. Results from simple formulae from earlier literature are also reported for reference. Section 5 presents a comparison between the model and a fully coupled FE-BE solution implemented in a commercial software. Final conclusions are given in Section 6.

\section{Vibroacoustic model for flat plates in a sound field}

Figure 1(a) shows a generic set-up and convention for a rectangular plate excited by an incident harmonic acoustic plane wave, while Figure 1(b) introduces the set-up and convention for the reradiated sound. An infinite rigid baffle is assumed elsewhere in the $x-y$ plane. The structure is analysed in the rectangular coordinate system $(x, y, z)$ and the pressure field is analysed in the spherical coordinate system $(r, \theta, \phi)$. Plane waves are assumed in the analysis. The boundary conditions at the plate edges are assumed to be arbitrary in the formulation, but the final results present examples for simply-supported edges.

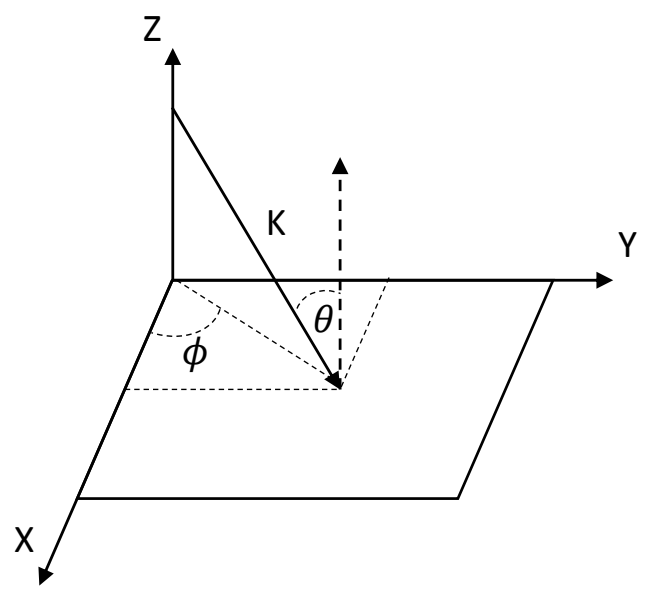

(a)

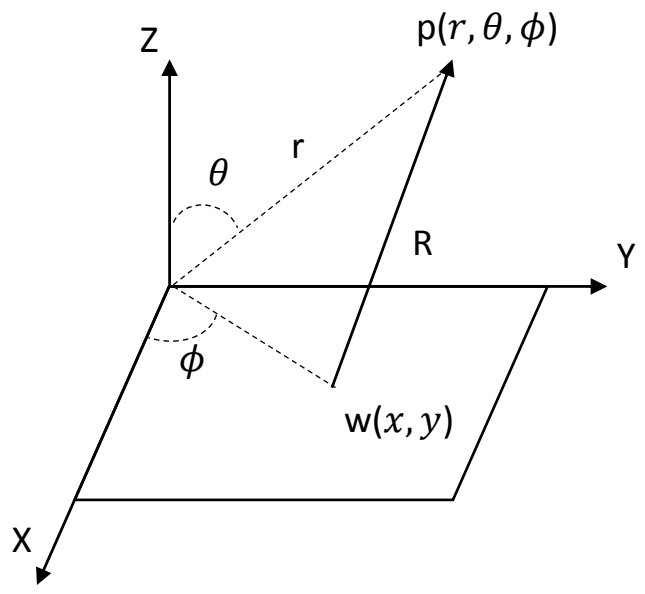

(b) 
Figure 1. An incident wave (a) onto and a radiated wave (b) from a rectangular plate in an otherwise infinite baffle. $k$ is the wave vector of the incident wave with the incident angle $\theta$ and the azimuthal angle $\phi$. R is the distance between the observation points for radiated pressure $p(\theta, \phi, r)$ and transverse vibration $w(x, y)$.

\subsection{The dynamic response for incident plane waves}

Using the expansion theorem [17], the displacement response of the plate can be written as a superposition of the normal modes $\psi_{n}(x, y)$ multiplied by the corresponding time-dependent modal displacement $q_{n}(t)$. Hence

$$
w(x, y, t)=\sum_{n=1}^{\infty} \psi_{n}(x, y) q_{n}(t)
$$

For harmonic excitation, the modal displacement can be expressed as

$$
q_{n}(\omega)=\frac{p_{0} a b j_{n}(\omega)}{Z_{n}(\omega)}
$$

where $Z_{n}(\omega)$ is the dynamic stiffness and $j_{n}$ is the JAF. The JAF can be evaluated from Eq. ( 1 ) as

$$
j_{n}(\omega)=\frac{1}{a b} \int_{0}^{b} \int_{0}^{a} \psi_{n}(x, y) \mathrm{e}^{-i k(x \cos \phi \sin \theta+y \sin \phi \sin \theta)} \mathrm{d} x \mathrm{~d} y
$$

\subsection{Sound transmission for incident plane waves}

The sound power radiated from the rear side of the plate can be obtained by integrating the far-field acoustic intensity over a hemisphere with radius $r$. For harmonic response, it can be written as

$$
W(\omega)=\frac{r^{2}}{2 \rho_{0} c} \int_{0}^{2 \pi} \int_{0}^{\frac{\pi}{2}}|p(r, \theta, \phi, \omega)|^{2} \sin \theta \mathrm{d} \theta \mathrm{d} \phi,
$$

where $\rho_{0}$ is the air density and $c$ is the sound speed in air. For a baffled plate coupled with the rear acoustic domain, this is equal to the transmitted sound power.

The incident sound power from the plane wave is

$$
W^{i}(\theta, \phi)=\frac{a b p_{0}^{2} \cos \theta}{2 \rho_{0} c}
$$

The sound power transmission coefficient $\tau$ is defined as [4] the ratio of transmitted to incident sound powers. Hence, 


$$
\tau(\theta, \phi)=\frac{4 r^{2}}{a b p_{0}^{2} \cos \theta} \int_{0}^{\frac{\pi}{2}} \int_{0}^{\frac{\pi}{2}}|p(\theta, \phi, r, \omega)|^{2} \sin \theta \mathrm{d} \theta \mathrm{d} \phi
$$

\subsection{Joint Acceptance Function (JAF) and modal radiation efficiency}

In order to obtain expressions for the radiation damping and for the vibroacoustic response of plates in diffuse field, it is convenient to relate the JAF with the modal radiation efficiency.

The radiated pressure can be calculated for the baffled plate using the Rayleigh integral [18]

$$
p(r, \theta, \phi, \omega)=\frac{-\omega^{2} \rho_{0}}{2 \pi R} \int_{0}^{b} \int_{0}^{a} w(x, y, \omega) \mathrm{e}^{-i k R} \mathrm{~d} x \mathrm{~d} y .
$$

Replacing the displacement using Eq. ( 1 ) and using a far-field approximation considering $r \gg a$ and $r \gg b$, therefore

$$
p(r, \theta, \phi, \omega) \approx-\frac{\omega^{2} \rho_{0} \mathrm{e}^{-i k r}}{2 \pi r} \sum_{n=1}^{\infty} q_{n} \int_{0}^{b} \int_{0}^{a} \psi_{n}(x, y) \mathrm{e}^{i k(x \cos \phi \sin \theta+y \sin \phi \sin \theta)} \mathrm{d} x \mathrm{~d} y .
$$

The double integral in Eq. ( 10 ) can be related to the complex conjugate of the JAF in Eq. ( 5 ). Hence the radiated pressure due to the $n^{\text {th }}$ mode can be written as

$$
p_{n}(r, \theta, \phi, \omega)=-\frac{\omega^{2} \rho_{0} a b \mathrm{e}^{-i k r}}{2 \pi r} q_{n} j_{n}^{*}(\theta, \phi, \omega)
$$

Substituting Eq. ( 11 ) into Eq. ( 10 ) and Eq. ( 6 ), the total sound power radiated from one side of the plate can then be expressed as,

$$
W(\omega)=\frac{\omega^{4} \rho_{0} a^{2} b^{2}}{2 \pi^{2} c}\left(\sum_{n=1}^{\infty} q_{n}^{2} \int_{0}^{\pi / 2} \int_{0}^{\pi / 2}\left|j_{n}\right|^{2} \sin \theta \mathrm{d} \theta \mathrm{d} \phi+\sum_{\substack{n=1 \\ u=1}}^{\infty} q_{n} q_{u}^{*} \int_{0}^{\pi / 2} \int_{0}^{\pi / 2} j_{n} j_{u}^{*} \sin \theta \mathrm{d} \theta \mathrm{d} \phi\right) .
$$

The first summation inside the brackets gives the radiated power associated with each individual normal mode. The second summation represents the modal cross terms. If these modal cross terms are neglected, the acoustic power radiated by the $n^{\text {th }}$ mode can be obtained by substituting Eq. (11) into Eq. ( 6 ), i.e.

$$
W_{n}(\omega)=\frac{\omega^{4} \rho_{0} a^{2} b^{2}}{2 \pi^{2} c} q_{n}^{2} \int_{0}^{\pi / 2} \int_{0}^{\pi / 2}\left|j_{n}(\theta, \phi, \omega)\right|^{2} \sin \theta \mathrm{d} \theta \mathrm{d} \phi
$$

The time averaged and spatial-averaged mean square normal velocity $\left\langle\overline{v_{n}^{2}}\right\rangle$ due to $n^{\text {th }}$ mode can be expressed as 


$$
\left\langle\overline{v_{n}^{2}}\right\rangle=\frac{\omega^{2} q_{n}^{2}}{2 a b} \int_{0}^{b} \int_{0}^{a} \psi_{n}^{2}(x, y) \mathrm{d} x \mathrm{~d} y
$$

Substituting Eq. ( 13 ) and Eq. ( 14 ) into Eq. ( 2 ), the relationship between the JAF and the modal radiation efficiency can be obtained as

$$
\sigma_{n}(\omega)=\left(\frac{k a b}{\pi}\right)^{2} \frac{\int_{0}^{\frac{\pi}{2}} \int_{0}^{\frac{\pi}{2}}\left|j_{n}\right|^{2} \sin \theta \mathrm{d} \theta \mathrm{d} \phi}{\int_{0}^{b} \int_{0}^{a} \psi_{n}^{2}(x, y) \mathrm{d} x \mathrm{~d} y}
$$

This double integral in the numerator of the above expression has been evaluated for a simply supported rectangular plate by Wallace [12]. A numerical solution is required when dealing with the other boundary conditions or stiffened plates.

\subsection{Vibroacoustic coupling}

The modal radiation damping and the added mass of the air should be considered when a fully coupled response is to be calculated.

The radiation damping loss factor for the $n^{\text {th }}$ mode is defined as [4]

$$
\eta_{n}^{r}(\omega)=\frac{W_{n}(\omega)}{\omega_{n} \bar{E}_{n}}
$$

where $\bar{E}_{n}$ is the time averaged vibrational energy associated to the $n^{\text {th }}$ mode, i.e. for a rectangular non-uniform plate

$$
\bar{E}_{n}=\int_{0}^{b} \int_{0}^{a} m_{A}(x, y) \overline{v_{n}^{2}} \mathrm{~d} x \mathrm{~d} y
$$

where $m_{A}(x, y)$ is mass distribution over the plate surface and has units of $\mathrm{kgm}^{-2}$.

Substituting Eq. ( 13 ) and Eq. ( 17 ) into Eq. ( 16 ), the modal radiation damping loss factor can be expressed as

$$
\eta_{n}^{r}=\frac{\rho_{0} c}{\omega_{n} M_{n}} \sigma_{n} \int_{0}^{b} \int_{0}^{a} \psi_{n}^{2}(x, y) \mathrm{d} x \mathrm{~d} y
$$

where $M_{n}$ is the modal mass given by $\int_{0}^{b} \int_{0}^{a} m_{A}(x, y) \psi_{n}^{2}(x, y) \mathrm{d} x \mathrm{~d} y$. 
The added-mass effect of the surrounding fluid is generally very small in the case of air. This can be neglected for all modes except the fundamental, for which it can be approximated from the reactance of the radiation impedance of a piston which vibrates with an equivalent volume velocity [4], i.e.

$$
a b\left\langle\overline{v_{1}^{2}}\right\rangle=\pi r_{e}^{2} \overline{v_{1}^{2}}
$$

where $r_{e}$ is the equivalent radius of the piston. Here the subscript 1 is used instead of $n$ to indicate that Eq. ( 19 ) is only valid for the fundamental mode.

The added mass of the air for a piston is $\frac{8}{3} \rho_{0} r_{e}^{3}$ [4]. The added mass of the air for the first vibration mode of the plate is then expressed as

$$
M_{1}^{r}=\frac{8}{3} \rho_{0}\left(\frac{\int_{0}^{b} \int_{0}^{a} \psi_{1}^{2}(x, y) \mathrm{d} x \mathrm{~d} y}{\pi}\right)^{\frac{3}{2}}
$$

As the modal stiffness is not changed, the first natural frequency becomes

$$
\omega_{1}^{\prime}=\omega_{1} \sqrt{\frac{M_{1}}{M_{1}+M_{1}^{r}}},
$$

where $M_{1}$ is the modal mass of the fundamental plate mode.

For the higher order modes the added mass can be approximated by $\frac{\rho_{0}}{k_{p}}$ for the case of $k_{p} \gg k$, where $k_{p}$ is the underlying free bending wavenumber of the plate material[4]. However, this is neglected in the present formulation.

\subsection{Vibroacoustic response for diffuse fields}

The diffuse sound field can be regarded as a superposition of harmonic plane waves with random phases and with equal amplitude $p_{0}$. These are incident on the baffled plate from all possible directions over one side, i.e. $\theta \in\left(0, \frac{\pi}{2}\right), \phi \in(0,2 \pi)[19]$.

The displacement response $w(x, y, \omega)$ on the plate surface to the diffuse field excitation can then be obtained by combining the response $w_{s}(x, y, \omega)$ to each plane wave $s$. As these plane waves are combined in a random manner, the individual displacement $w_{1}, w_{2}, \ldots, w_{s} \ldots$ are uncorrelated and the total mean square response $\overline{w^{2}}$ is found by adding up the mean square values of the individual responses: 


$$
\overline{w^{2}}=\left(2 a b p_{0}\right)^{2} \lim _{S \rightarrow \infty} \frac{1}{S} \sum_{S=1}^{S}\left(\sum_{n=1}^{\infty} \frac{\psi_{n}^{2}\left|j_{n, s}\right|^{2}}{Z_{n}^{2}}+\sum_{\substack{n=1 \\ u=1}}^{\infty} \frac{\psi_{n} \psi_{u} j_{n, s} j_{u, S}^{*}}{Z_{n} Z_{u}^{*}}\right)
$$

where $S$ is the total number of plane waves used to represent a diffuse field and $Z_{n}=$ $M_{n}^{2}\left[-\omega^{2}+\omega_{n}^{2}+i\left(\eta+\eta_{n}^{r}\right) \omega_{n}^{2}\right]$ is the modal dynamic stiffness of the plate. The first summation inside the brackets gives the sum of the mean square response in the individual normal modes, i.e. the direct mean square response. The second summation represents the cross terms.

If the cross term contribution is neglected, the value of the summation outside of the brackets in Eq. (21) can be evaluated by using the hemispherical model. The area element of an infinitesimal segment for the hemisphere is $r^{2} \sin \theta \Delta \phi \Delta \theta$. As the segment becomes small enough, the number of plane waves passing through it can be approximated as $\left(\frac{S}{2 \pi r^{2}}\right) r^{2} \sin \theta \Delta \phi \Delta \theta$, each of which is associated with the same value $\left|j_{n, s}(\theta, \phi, \omega)\right|^{2}$. Therefore, Eq. ( 22 ) can be simplified as

$$
\overline{w^{2}} \approx\left(2 a b p_{0}\right)^{2} \sum_{n=1}^{\infty} \frac{\psi_{n}^{2}(x, y)}{Z_{n}^{2}(\omega)}\left(\frac{2}{\pi} \int_{0}^{\frac{\pi}{2}} \int_{0}^{\frac{\pi}{2}}\left|j_{n}\right|^{2} \sin \theta \mathrm{d} \phi \mathrm{d} \theta\right) .
$$

This equation replaces a discrete summation over $\mathrm{S}$ in Eq. ( 22 ) by an integral of incident and azimuthal angles, which has the same form appearing in Eq. ( 15 ).Substituting Eq. ( 15 ) into the above expression, the mean square response to the diffuse sound field can be directly related to the modal radiation efficiency as,

$$
\overline{w^{2}} \approx 8 p_{0}^{2} \frac{\pi c^{2}}{\omega^{2}} \sum_{n=1}^{\infty} \frac{\psi_{n}^{2}(x, y)}{Z_{n}^{2}(\omega)} \sigma_{n}(\omega) \int_{0}^{b} \int_{0}^{a} \psi_{n}^{2}(x, y) \mathrm{d} x \mathrm{~d} y
$$

\subsection{Sound transmission for diffuse field}

The sound transmission coefficient of a diffuse field is

$$
\tau_{d}=\frac{\int_{0}^{2 \pi} \int_{0}^{\frac{\pi}{2}} \tau(\theta, \phi) \sin \theta \cos \theta \mathrm{d} \theta \mathrm{d} \phi}{\int_{0}^{2 \pi} \int_{0}^{\frac{\pi}{2}} \sin \theta \cos \theta \mathrm{d} \theta \mathrm{d} \phi} .
$$


Substituting Eq. ( 8 ) and Eq. ( 15 ) into Eq. ( 25 ), this can be expressed as

$$
\tau_{d}=\frac{16 \pi \rho_{0}^{2} c^{4}}{a b} \sum_{n=1}^{\infty} \frac{\sigma_{n}^{2}}{Z_{n}^{2}} \int_{0}^{b} \int_{0}^{a} \psi_{n}^{2}(x, y) \mathrm{d} x \mathrm{~d} y
$$

This is often presented using the sound Transmission Loss (TL): $T L=10 \log _{10}\left(\frac{1}{\tau_{d}}\right)$.

The expressions for the plate's displacement response (Eq. ( 24$)$ ) and the sound transmission coefficient (Eq. ( 26 )) show that the fully coupled vibroacoustic response of structure to a diffuse field can be evaluated by knowing the mode shapes, the natural frequencies, the damping and the modal radiation efficiency. The first two quantities can be obtained from a free vibration analysis by using either analytical or numerical approaches, while the modal radiation efficiencies can be evaluated from Eq. ( 15 ).

\section{Structural model}

The vibroacoustic study presented in Section 2 relies on knowing the modal properties of the plate. Different approaches exist to evaluate the free vibration of stiffened plates. Analytical models, such as the assumed-modes approach, have been presented $[16,20]$ and they are valid within the assumption of light stiffeners; usually the in-plane motion of the plate is neglected and the platestiffener interface is treated as a line. Alternatively, a structural in vacuo FE model can provide more accurate results as it usually involves less simplification than an analytical model representation. For example, the in-plane motion of the plate is retained using shell elements, or laminate shell elements for composite plates, with the stiffeners being modelled as beams, shells or solids depending on the configuration and geometry (i.e. straight or curvilinear). This may result in an increased computational cost compared to an analytical one, but the free vibration analysis of the FE model can still solved within a reasonable time to cover the modes up to a certain frequency range of interest. Subsequently, the integral expressions for the vibroacoustic response (section 2) can be evaluated using a numerical discretisation of the integrals over the structural domains. 
Figure 2 shows the geometry of the orthogonally stiffened, rectangular plate adopted as an example in this study. The plate is ribbed orthogonally by two prismatic stiffeners parallel to the $y$-axis and two prismatic stiffeners parallel to the $x$-axis, as illustrated in Figure 2 (a). The cross section of the stiffeners is depicted in Figure 2 (b).

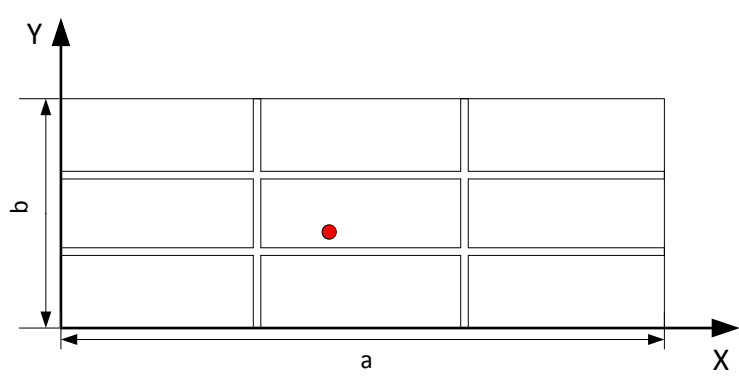

(a)

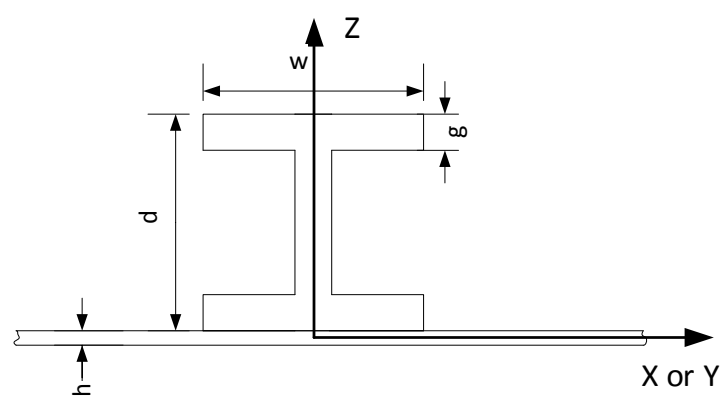

(b)

Figure 2. Geometry of an orthogonally stiffened, rectangular plates. (a) stiffener layout: two evenly spaced stiffeners in the $x$ and $y$ directions, respectively; (b) stiffener detail: I section.

Table 1 lists the geometry and material properties used in the simulation. Damping ratios of aircraft panels are adopted and these are usually in the $0.5 \%-2 \%$ range [21]. Therefore, a constant value of structural damping loss factor of $1 \%$ is assumed in this work. Simply supported boundary conditions are adopted in this work, but these could be changed in the structural model as required.

Table 1. The structural parameters for the stiffened plates.

\begin{tabular}{|c|c|c|c|c|c|}
\hline \multicolumn{2}{|c|}{ Plate (unit: $\mathrm{mm}$ ) } & \multicolumn{2}{l|}{ Stiffener (unit: $\mathrm{mm}$ ) } & \multicolumn{2}{l|}{ Material (aluminium) } \\
\hline$a$, length & 2400 & $w$, width & 30 & $E$, Young's modulus (GPa) & 71 \\
\hline$b$, width & 900 & $d$, depth & 30 & $\rho$, density $\left(\mathrm{kg} / \mathrm{m}^{3}\right)$ & 2700 \\
\hline$h$, thickness & 2 & $g$, thickness & 5 & $v$, Poisson's ratio & 0.3 \\
\hline
\end{tabular}

It can be seen from the structural dimensions that the thickness of the plate is 15 times smaller than the height of the stiffeners. This may result in a significant coupling between the out-of-plane and inplane displacements of plates for the bending motion, which is neglected in the analytical models available in the literature. The emphasis of the current research is on the vibroacoustic behaviour of 
stiffened plate and the generic configurations of the stiffeners may not necessarily meet the full assumptions of light stiffeners required in analytical models [20].

In order to evaluate possible approaches to predict the free vibration of stiffened plates, the mode shapes and natural frequencies calculated from an analytical and a finite element model are compared. The analytical model is the one given and detailed by Dozio [20]. The MATLAB code was implemented with the number of trial functions selected by verification of the solution's convergence over the frequency range up to $1500 \mathrm{~Hz}$; this corresponds to the first 420 modes. The Finite Element model was developed in the 'Structural mechanics' module of COMSOL [22]. The plate was represented with quadratic shell elements and the stiffeners with cubic beam elements with 'I-profile' cross section. The beams were then coupled to the plate in the setting of 'Shell-Beam Connection' in the 'Multiphysics' module. The mesh size was selected by verification of the solution's convergence over a frequency range up to $1500 \mathrm{~Hz}$, including 431 modes. The total number of elements required in the model was 6354. (For the Finite Element model see also Section 5.2 below.)

Figure 3 shows the comparison of the modal properties in terms of the Modal Assurance Criterion (MAC) [23], for the mode shapes, and the scatter plots for the first 20 natural frequencies.

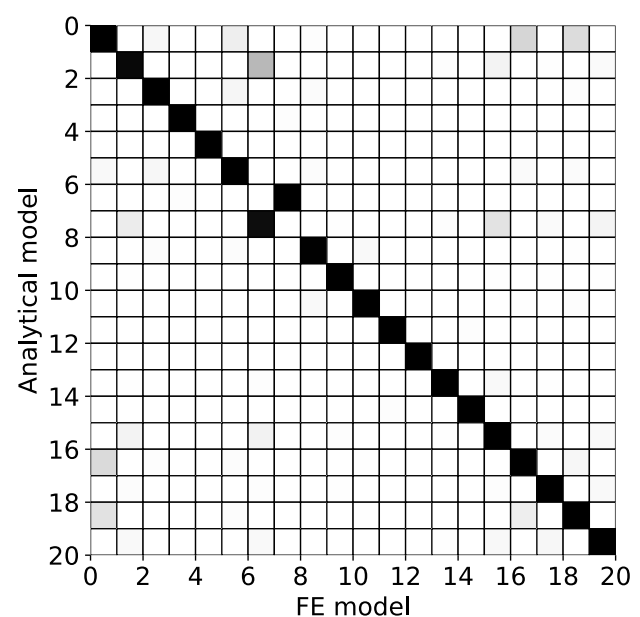

(a)
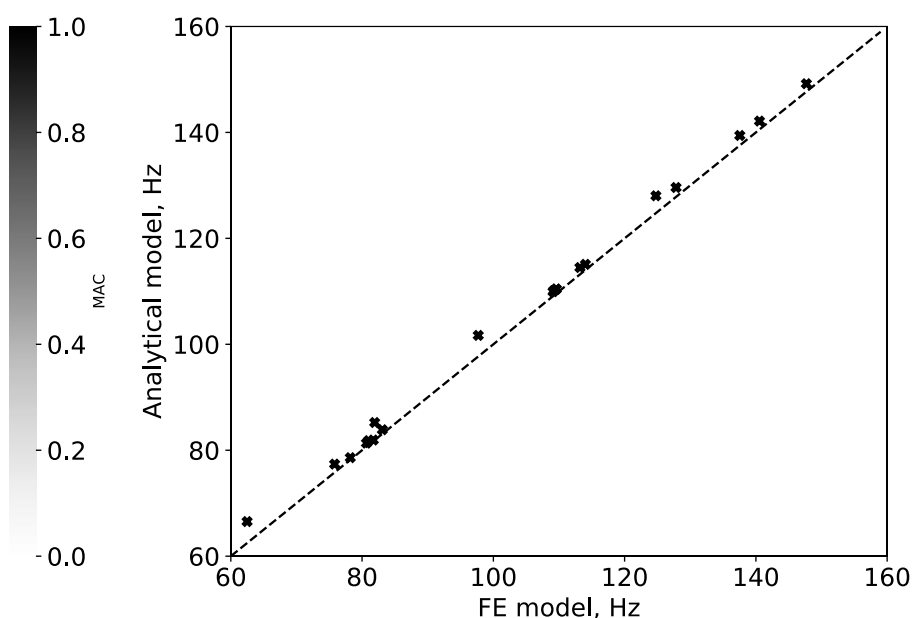

(b)

Figure 3. Comparison of modal properties between a FE model and an analytical model for the lowest 20 modes of the stiffened plate. (a) MAC plot; (b) Scatter plot. 
The analytical model provides a good prediction for the natural frequencies and mode shapes for the first 20 modes. However, the natural frequencies predicted by the analytical model tend to be higher than those calculated by FE and this is due to neglecting the deformation and hence strain energy associated with the in-plane motion of the plate. This also applies to the first mode, which is known to be typically the major contributor to the response of a flat plate in an acoustic field [24]. Although the mode shape agreement for the two models of this mode is high as seen in the MAC value, the difference in the fundamental natural frequency, i.e. the first point in the scatter plot Figure 3(b), leads to a shift in the corresponding resonance. In addition, the MAC for higher frequency modes show larger but still acceptable differences between the analytical and the numerical results.

For the purpose of performing parametric evaluations, the analytical model is subsequently used to generate the modal basis behind the calculations reported next in Section 4. In Section 5, the FE structural model is used, coupled with an acoustic domain, to validate the vibroacoustic calculations introduced in Section 2. In order to align the modal basis used as an input in the proposed modal model with the numerical model, the modal basis obtained from the FE model is then preferred to the analytical one, although the modes are generally still in good agreement.

\section{Numerical results}

\subsection{Dynamic response}

The dynamic responses of the plate subjected to acoustic excitations are given in terms of the transverse displacement at an arbitrary reference point. The point position is at $(1.06,0.38) \mathrm{m}$ and it is highlighted in Figure 2 (a). In addition, the spatially-averaged displacement is also evaluated. The sound pressure level (SPL) of the incident sound field is $94 \mathrm{~dB}$ for all the cases evaluated. The diffuse field is constructed by 603 incident plane wave above the plate surface. The method for calculating the direction of these waves is taken directly from the reference [6]. The amplitude of each individual wave is $1 \mathrm{~Pa}$ and the phase of them is taken from a uniform random distribution between $\pm \pi$ with zero mean value. The diffuse field pressure is obtained by adding up these waves then divided by the 
square root of the number of waves to ensure that the limit remains finite as the number of contributing waves becomes infinite [7]. A large number of numerically simulated samples of the diffuse field is also required in order to produce a good approximation to the theoretical spatial pressure correlation. In this study, the mean square error between the averaged spatial pressure correlation of the simulated diffuse field and that of the theoretical diffuse field, i.e. $\frac{\sin k x}{k x}$, was calculated. The mean square error, as measured for the spatial correlation, was less than $4 \%$ when averaged over 100 different realizations of the diffuse field for a Helmholtz number, $k x$, less than 44 . The error can be reduced further with an increased number of samples. However, a higher accuracy of the reproduced diffuse field was unnecessary, so 100 samples were used in this study without incurring a significant computational cost.

The comparison of the results for the unstiffened and stiffened plate are given to highlight the effect of the stiffeners. These results are given as the transfer function between the transverse displacement of the plates and the unit magnitude pressure input for the sound field. The displacement is normalised by the plate thickness. The frequency range is up to $1000 \mathrm{~Hz}$, with a frequency resolution of 200 points per decade, and the frequency axis is normalised by the lowest natural frequency of the corresponding structure, which is identified as $f / f_{n=1}$ for the transfer function results in Section 4 . The analytical model introduced in section 2 is used here to evaluate the effect of different excitation types, the effect of vibroacoustic coupling and that of the modal cross terms.

\subsubsection{Plane wave and diffuse field excitation}

Figure 4 shows the responses to three different acoustic excitations. The results are presented in the presence of vibroacoustic coupling and including the modal cross terms. 


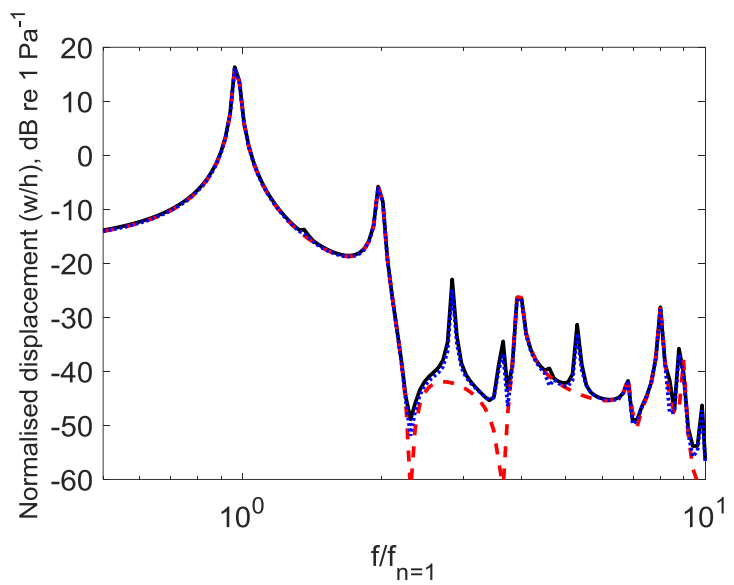

(a)

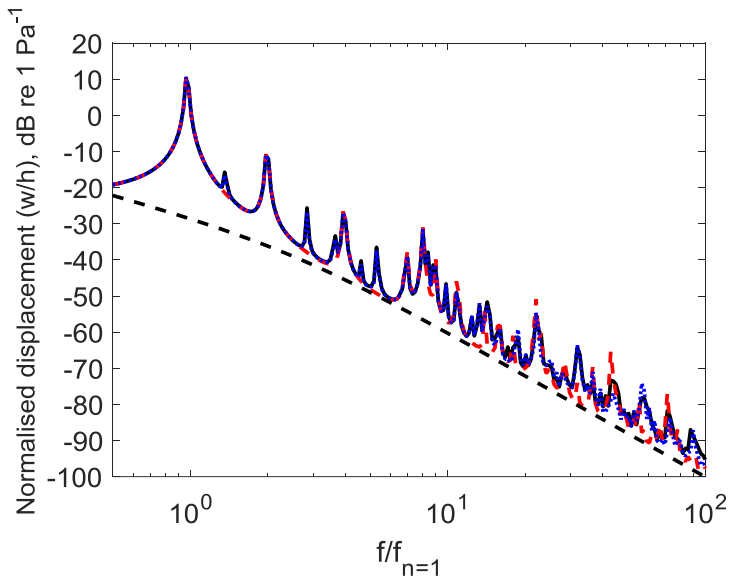

(c)

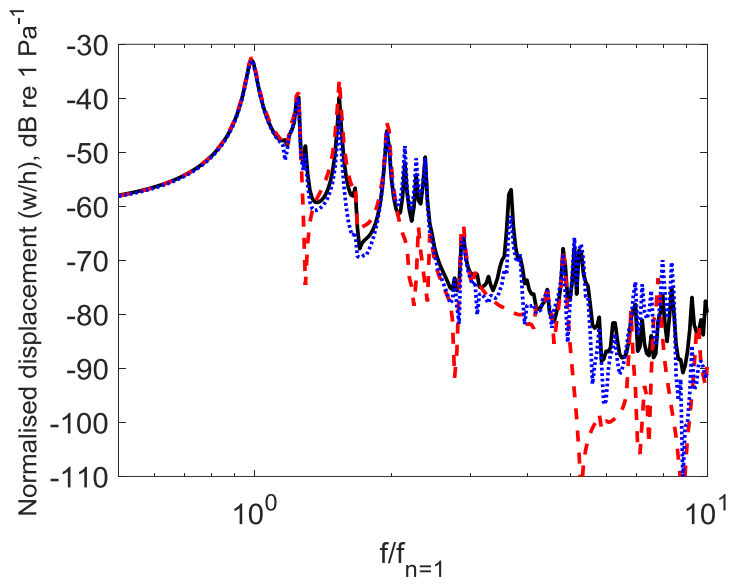

(b)

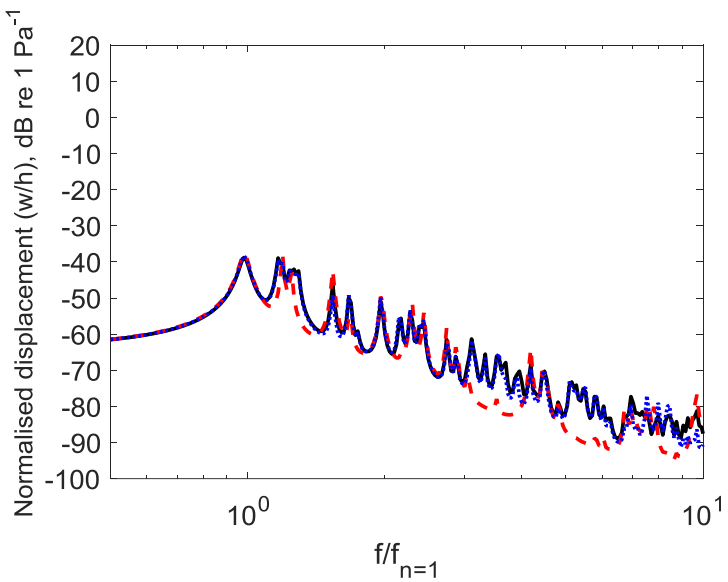

(d)

Figure 4. The normalized transverse displacement transfer function for different acoustic excitation: diffuse sound field (black solid lines), normal incident plane wave (red dashed lines) and oblique incident plane wave $(\theta, \phi)=\left(\frac{\pi}{4}, \frac{\pi}{4}\right)$ (blue dotted lines). (a) reference point response of the unstiffened plate. (b) reference point response of the stiffened plate. (c) spatially-averaged response of the unstiffened plate including a black dashed line calculated from the Liamshev's planewave response theory for an oblique incident plane wave. (d) spatially-averaged response of the stiffened plate.

The results for the reference point in Figure 4 (a) and (b) show that the responses are similar for the three different acoustic excitations at the lower frequencies, where the structural wavelength is much smaller compared to the acoustic wavelength. This is because the JAFs for both the plane waves and the diffuse field are similar to the normal incidence case, i.e. only the symmetric modes can be excited. In the high frequency range, the number of resonances significantly responding decreases for normal 
plane wave excitation compared to the other two sound fields. For the unstiffened plate case in Figure 4 (a), eight resonances can be clearly observed for both the diffuse field and the oblique plane wave excitation, while only four of them are excited by the normal incidence wave. A plot of the corresponding mode shapes, which is not given here for brevity, shows that these non-responsive modes are all antisymmetric modes about one or two axes, and this corresponds to JAFs equal to zero. While this is true for the unstiffened plate with fully simply supported edges, for a more general case, e.g. a mixture of clamped, elastically supported etc., which are not the same on opposite edges, the modes will not be purely symmetric or antisymmetric. Thus the number of excited modes will increase. The difference in the number of acoustically excited modes for the different acoustic fields is smaller for the stiffened plate case in Figure 4 (b). This is because the orthogonal stiffeners lead to a fewer number of antisymmetric modes. However, the response of the stiffened plate excited by a normal plane wave tends to be lower than that due to an oblique plane wave or the diffuse sound field excitation, both in terms of the number of excited modes and the peak amplitudes.

The results for the spatially-averaged displacement presented in Figure 4 (c) and (d) are calculated for a rectangular grid of $46 \times 121$ points and confirm the main behaviour found at the reference point in terms of the response due to different excitation. For reference, the unstiffened plate is also compared with the result calculated from Liamshev's plane-wave response theory [4]. This is only valid for an unstiffened, infinite plate excited by an oblique plane wave, which is set to be $(\theta, \phi)=$ $\left(\frac{\pi}{4}, \frac{\pi}{4}\right)$.The comparison with Liamshev's formulation is satisfactory for $\frac{f}{f_{1}}>10$, although the latter tends to underestimate the response and follows the minima.

\subsubsection{Effect of vibroacoustic coupling}

Figure 5 shows the effect of the vibroacoustic coupling for a broadband diffuse field. The modal crossterms are included in this analysis. 


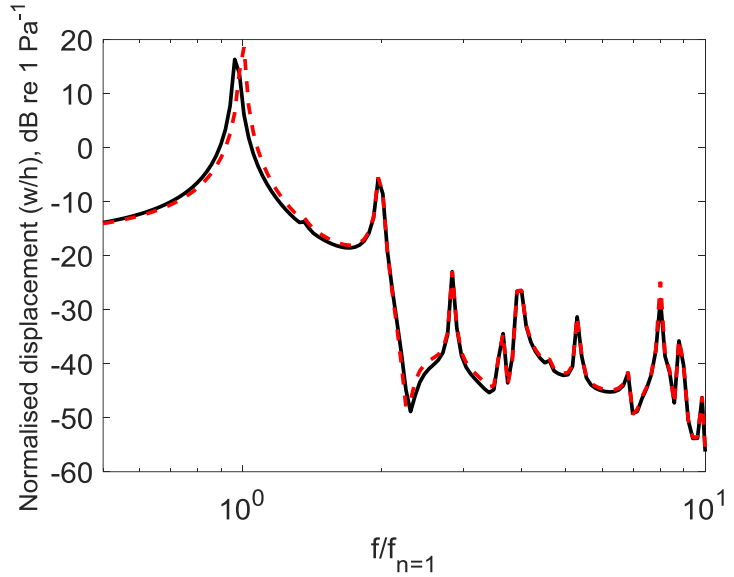

(a)

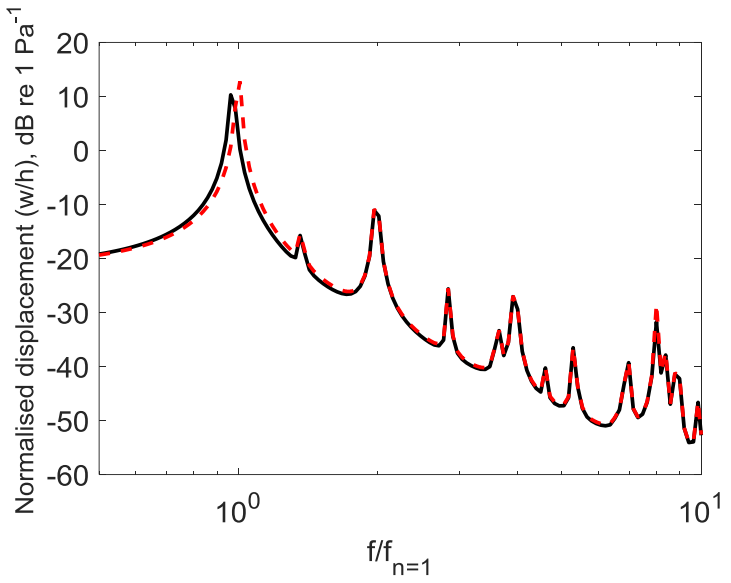

(c)

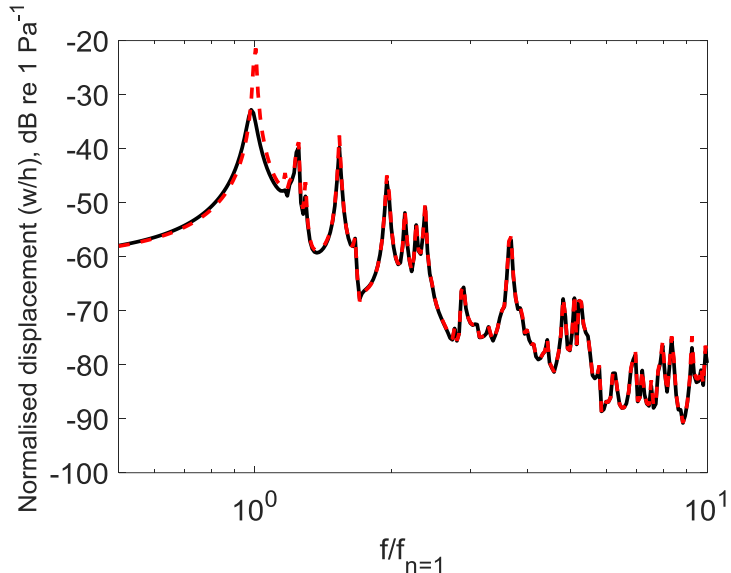

(b)

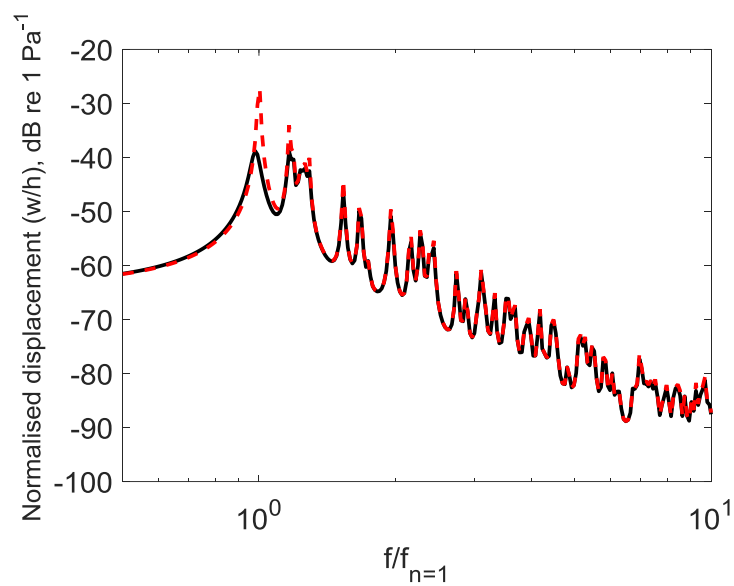

(d)

Figure 5. The effect of the vibroacoustic coupling on the transfer function of the normalized transverse displacement. Black solid lines are the fully coupled response and the red dashed lines are the uncoupled response. (a) reference point response of an unstiffened plate. (b) reference point response of a stiffened plate. (c) spatially-averaged response of unstiffened plate. (d) spatially-averaged response of stiffened plate.

From the comparison between the uncoupled and the fully coupled response at the reference point, there is a significant reduction in the level of the dominant resonance, about $11.4 \mathrm{~dB}$, for the stiffened plate in Figure 5 (b). While a smaller difference, of about $2.3 \mathrm{~dB}$, is observed for the unstiffened plate in Figure 5 (a). The radiation damping calculated for the stiffened plate in Eq. ( 18 ) was found to be larger than that of the unstiffened plate by a factor of five. This can be explained by noticing that the 
stiffeners increase the fundamental natural frequency of the plate and hence the corresponding modal radiation ratio evaluated at this natural frequency increases as well, thus leading to a larger value of the radiation damping at this frequency. In addition, the stiffeners also cause the plate mode shapes to deviate from a sinusoidal form, which introduces lower wavenumber spatial vibration components that match better with the acoustic wavenumber and increases the modal radiation efficiency. For the structural configuration used in simulation, this effect is less significant than the effects due to the increase in the natural frequencies.

The spatially averaged response confirms again the main trend found for the single point. The vibroacoustic coupling for the case in Figure 6(d) reduces the amplitude of the first peak by $11.6 \mathrm{~dB}$ and makes the first 2 modes almost equally contribute to the overall response.

The vibroacoustic coupling effect for different stiffener configurations is also analysed here. In Figure 6 (a), four types of plates are used for comparison of different stiffener arrangements and these are studied for two different height of the stiffeners' cross section. The first is the one specified in Table 1 and the second one corresponds to reducing the former by $1 / 3$. Figure 6 (b) shows the dB difference of the spatially averaged RMS displacement evaluated by using the blocked pressure versus a full vibroacoustic coupling including re-radiation. To calculate the RMS, a broadband random noise with flat spectrum up to $1000 \mathrm{~Hz}$ has been assumed.

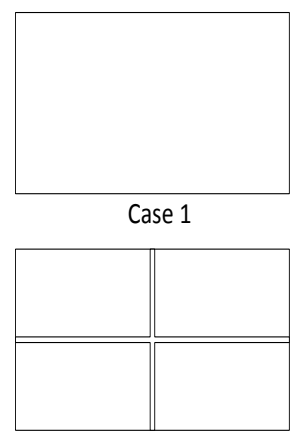

Case 3
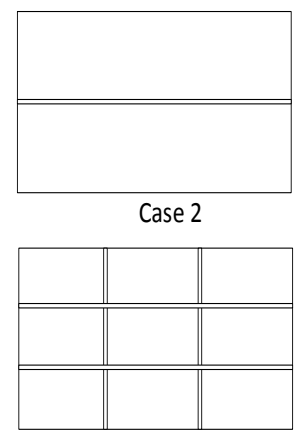

Case 4

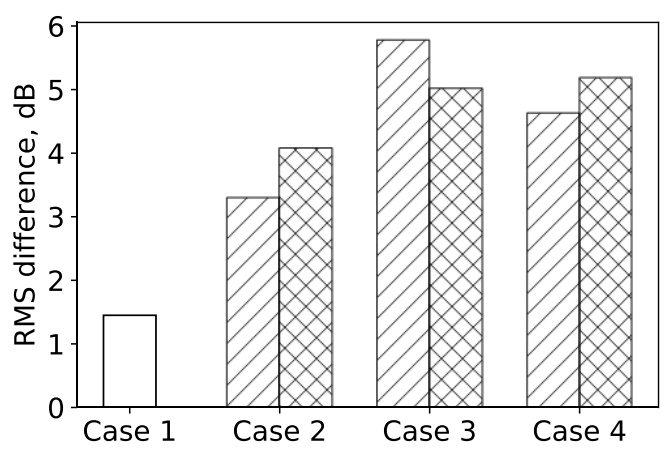

(a)

(b) 
Figure 6. Comparison of vibroacoustic coupling effect for different cases. (a) four types of plates. Case 1: unstiffened plate; Case 2: plate with $1 x$-wise stiffener; Case 3: plate with $1 x$-wise stiffener and $1 y$-wise stiffeners; Case 4: plate with $2 x$ wise stiffeners and $2 y$-wise stiffeners. (b) dB difference of the spatially-averaged RMS displacement for different cases using either a full vibroacoustic coupling or an uncoupled (blocked force) model.

The result show that, in general, the effect of vibroacoustic coupling increases as the number of stiffeners increases and that on average there is also an increase in this effect when the height of the stiffeners decreases. The results in Figure 6 are however representative of an average 'single-number' response and specific results may vary for different points on the plate.

The modal contributions to the total RMS response are also affected by the vibroacoustic coupling. For example, for the stiffened plate the contribution of the fundamental mode is $89 \%$ for the uncoupled (blocked force) response while it decreases to $63 \%$ for the fully coupled configuration. To obtain $99 \%$ of the total RMS for the stiffened plates (case 4), the required number of modes is 10 for the uncoupled response, and this is increased to 23 for the fully coupled response.

\subsubsection{Effect of modal cross-terms}

Figure 7 shows the effect of the cross terms on the response to a diffuse field and in the presence of vibroacoustic coupling. The black solid lines represent the response without the modal cross terms coupling and the red solid lines represent the response with the cross terms coupling included.

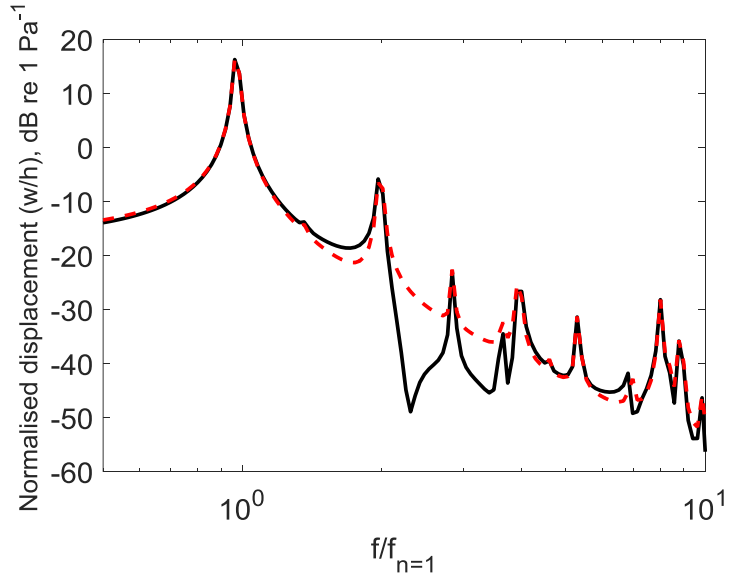

(a)

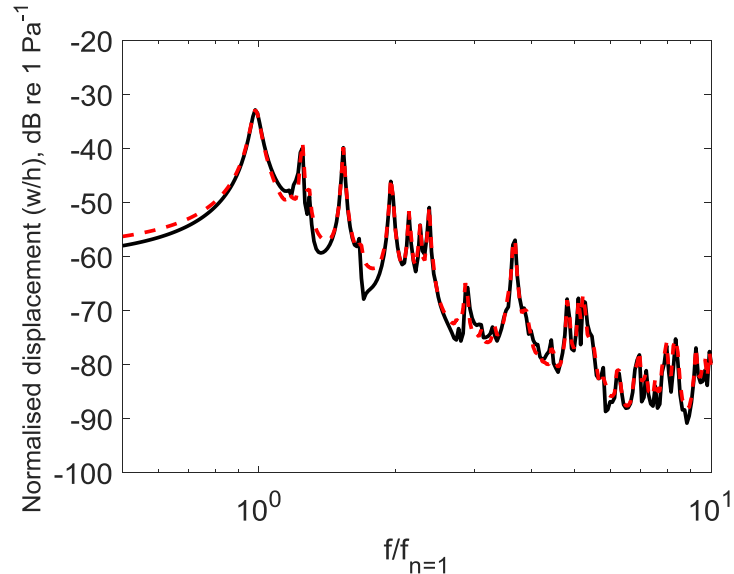

(b)

Figure 7. The effect of the cross-term modal contribution on the transfer function of the normalized displacement response of the reference point on the unstiffened (a) and the stiffened panels (b) excited by a diffuse field. Black solid lines: without cross-term modal contribution. Red dashed lines: with the modal cross-term contribution. 
The results show that the contribution from the modal cross terms is more significant at the lower frequencies and less significant at the higher frequencies for both the unstiffened and stiffened plates. This is because the modal cross terms mainly influence the non-resonant frequency regions. The cross term contributions, due to modal orthogonality, have no effect on the spatially averaged response as seen in Eq. ( 22 ). Although the results in Figure 5 and Figure 7 are for the diffuse sound field, the effects of the vibroacoustic coupling and the modal cross terms are similar for the other acoustic excitation types analysed. The corresponding results are not presented here.

\subsection{Sound transmission loss ( $T L)$}

\subsubsection{Plane wave and diffuse field excitations}

Figure 8 shows the TL for the three different acoustic excitations analysed above; the simulations are performed including the full vibroacoustic coupling. The modal cross terms are also included in these results.

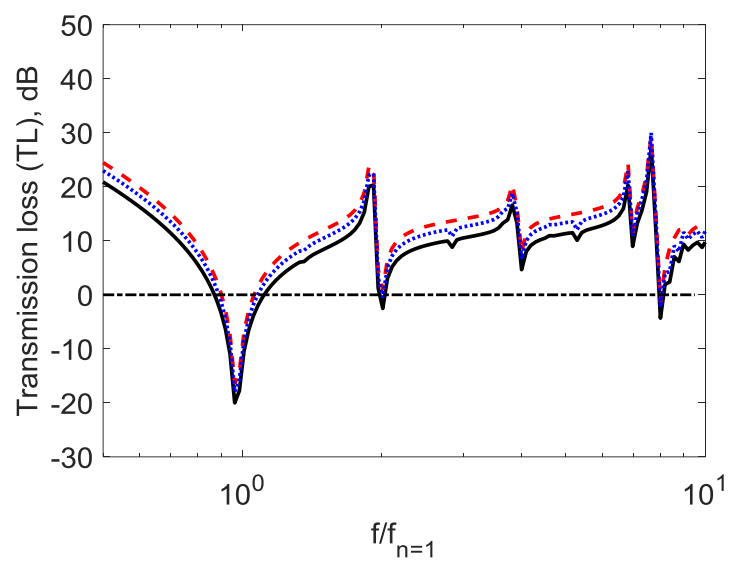

(a)

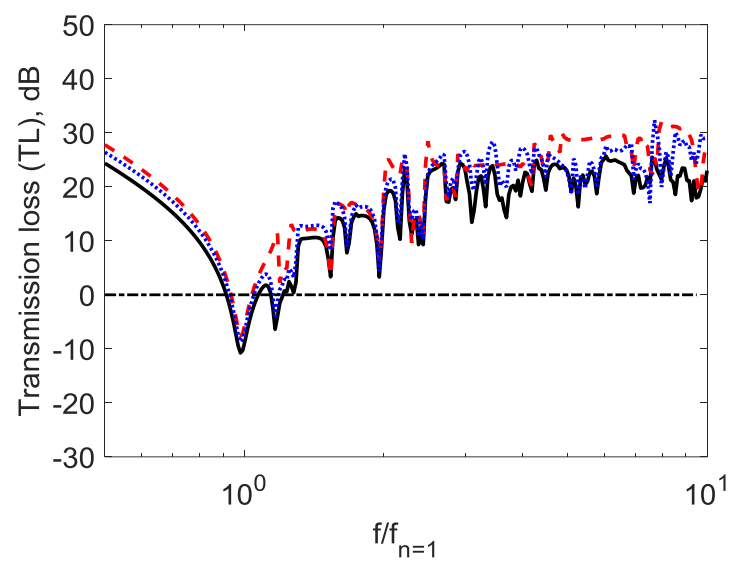

(b)

Figure 8. The effect of acoustic excitation on the TL of the unstiffened (a) and the stiffened panels (b). Black solid lines: an incident diffuse field. Red dashed lines: a normal incident plane wave. Blue dotted lines: an oblique incident plane wave at

$$
\text { an angle }(\theta, \phi)=\left(\frac{\pi}{4}, \frac{\pi}{4}\right) \text {. Horizontal dash-dot line: } 0 \mathrm{~dB} \text { line. }
$$

The TL results show that behaviour characterized by a stiffness-controlled region and an intermediate frequency region which are separated by the first plate resonance. The effect of different acoustic 
excitation types is the same in the stiffness-controlled region for both the unstiffened plate and the stiffened plate, i.e. the TL decreases by $1-2 \mathrm{~dB}$ from the normal to oblique to diffuse field incidence. The same behaviour is also extended to the intermediate frequency region for the unstiffened plate. Although the number of acoustically excited modes are different for the three types of acoustic excitation, only the symmetric modes can effectively radiate sound. This can be observed from the dips in the TL, i.e. plate resonances, in Figure 8 (a). Figure 8 (b) shows that although the response of stiffened plates differs from the unstiffened one, i.e. the number of dips present in the intermediate frequency region is significantly increased, the main differences in the TL for different acoustic fields are preserved. It is also worth noting that the TL is negative at some low-frequency plate resonances. This is a consequence of the normalization used for the incident sound power as explained by Thompson et al. in [25].

Analytical formulae for the normal incidence mass law, the diffuse incidence mass law, the field incidence mass law [4] and Sewell's diffuse field [26] are also presented in Figure 10 for comparison. They are valid for unstiffened plates and can approximate the behaviour of a finite plate in the high frequency range. Figure 9 shows the comparison of the results for the unstiffened plate subjected to both the normal incident plane wave and the diffuse field. Note that Sewell's formula used in the calculation is simplified from the original one (formula (54) in [26]) by neglecting the small correction terms. 


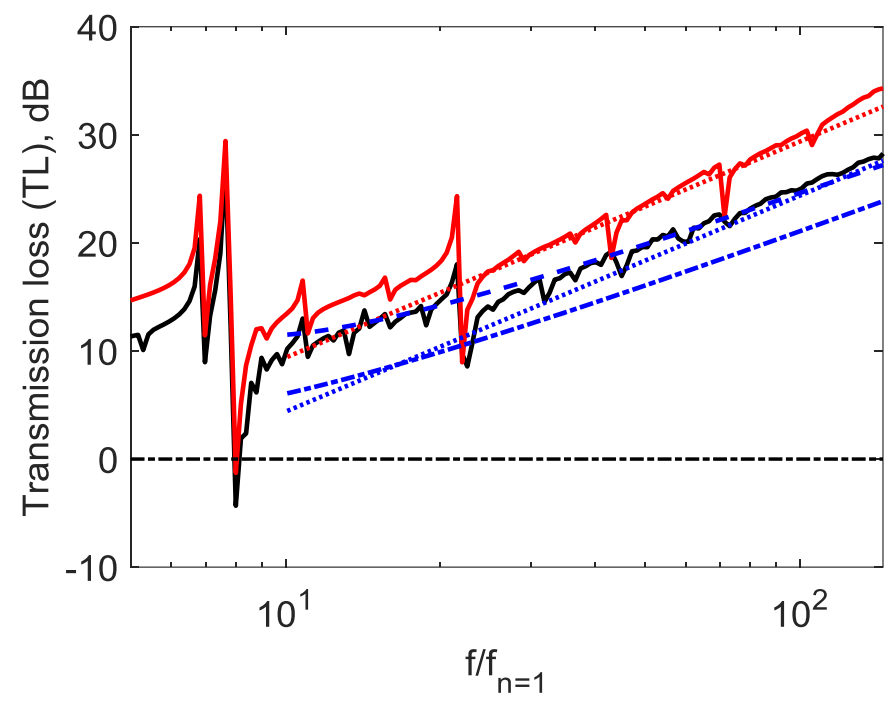

Figure 9. The comparison of TL results between the proposed model and the approximate formulae for the unstiffened plate subjected to a normal incident plane wave and a diffuse field excitation. Red, solid line: the proposed model subjected to normal incident plane wave. Black, solid line: the proposed model subjected to diffuse field. Red, dotted line: the normal incidence mass law. Blue, dash-dot line: the diffuse field mass law. Blue, dotted line: the field incidence mass law. Blue, dash line: Sewell's simplified formula. Horizontal dash-dot line: $0 \mathrm{~dB}$ line.

The results show a good agreement for a normal incident wave in the high-frequency range $\left(\frac{f}{f_{1}}>50\right)$. However, for the diffuse field results a significant difference in the TL is observed in comparison to the diffuse field mass law (blue, dash-dot line). The same phenomenon has been observed by Sewell, with the explanation that the finite plate cannot radiate well at high angles [4]. The agreement is improved with comparison to the field incidence mass law (blue, dotted line), which excludes the contribution of the high angles between $78^{\circ}-90^{\circ}$. A further improvement at the low frequencies is achieved in the comparison to Sewell's formula (blue, dashed line), which explicitly includes the effect of the finite plate dimensions.

\subsubsection{The effects of modal cross-terms}

Figure 10 shows the effect of the modal cross terms on the TL to a diffuse field and in the presence of vibroacoustic coupling. The black solid lines represent the TL including the modal cross terms and the red dashed lines represent the response with the cross terms excluded. 


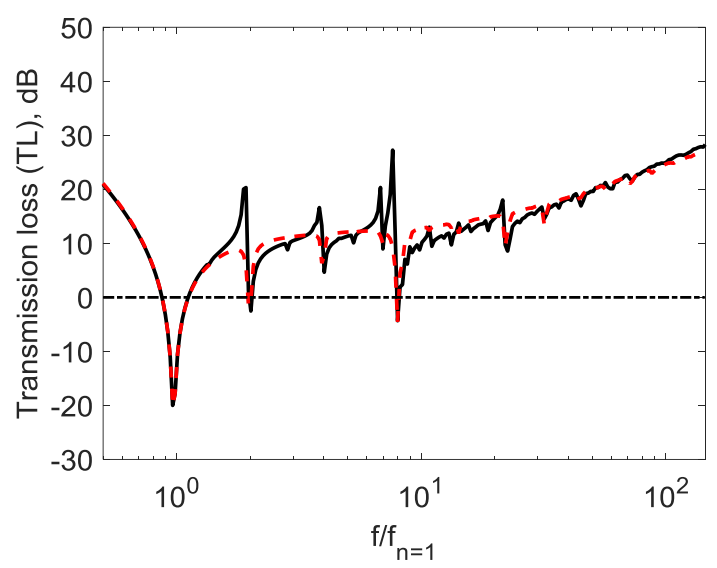

(a)

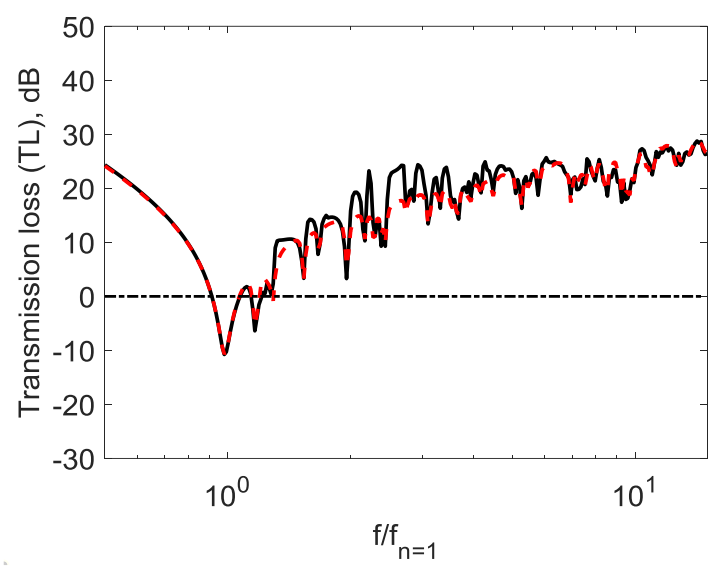

(b)

Figure 10. The effect of the cross-term modal contribution on the TL on the unstiffened (a) and the stiffened panels (b) excited by the diffuse field. Black solid lines: include cross-term modal contribution. Red dashed lines: without the crossterm modal contribution. Horizontal dash-dot line: $0 \mathrm{~dB}$ line.

The results show that the modal cross term contribution is on average small, but it can be significant in between resonances at some frequencies. The effect of full vibroacoustic coupling on the TL for diffuse field has been examined and found to give results consistent with that of the plate response in Figure $5(a)$ and (b), so it is not presented here.

\section{Comparison with a FE-BE model}

To validate the approach introduced in this work, the results obtained with the proposed model are compared with coupled FE-BE numerical simulations developed in COMSOL. For this comparison, the modal basis of the stiffened plate for the analytical modal model is obtained from the same structural model adopted in COMSOL for this validation. 431 modes are used in the modal summation which covers the frequency range up to $1500 \mathrm{~Hz}$.

Figure 11 (a) shows the meshed FE-BE model. The details of structural FEM has been given in Section

3. The plate FEM is embedded in the upper surface of a rigid cylinder which approximates an infinite baffle. The acoustic BEM was then developed in the 'Pressure acoustic, boundary elements' module to model the external air domain. Quadratic boundary elements were used to mesh the boundary between the external air domain and the baffled, stiffened plates, including the rigid cylinder. The 
structural FEM and the acoustic BEM are coupled by the 'Acoustic-structure boundary' in the 'Multiphysics' module.

To simulate the total pressure at the plate interface with the air domain, the sound field is divided into two parts. The first part comprises the incident and reflected pressures for a rigid surface, hence corresponding to a blocked pressure, while the second part consists of the pressure radiated from the vibrating plate [4], i.e.,

$$
p_{\text {total }}=p_{\text {incident }}+p_{\text {reflected }}+p_{\text {radiated }}=p_{\text {block }}+p_{\text {radiated }}
$$

To allow the same diffuse field pressure field to be used in the analytical model and in the numerical approach, the term $p_{\text {block }}$ is imported from the modal model of Section 2 into the coupled FE-BE model in COMSOL. The term $p_{\text {radiated }}$, is automatically included in the numerical simulation via FEBE coupling.

To minimise the effect of diffraction from the edges of the rigid cylinder, its radius was set to be at least half the largest acoustic wavelength. The mesh size of the boundary element was selected by verification of the solution's convergence over a frequency range up to $1000 \mathrm{~Hz}$. The coupled $\mathrm{FE}-\mathrm{BE}$ model was then solved in the frequency domain with an iterative solver. As with the analytical model, the radiated sound power above the plate surface is used to calculate the transmitted sound power to the other side of the plate. For harmonic excitation, the time-averaged acoustic intensity of the radiated sound field over the plate surface is evaluated using the equation $I=\frac{1}{2} \operatorname{Re}\left\{p_{\text {rad }} \times v^{*}\right\}$, where $v$ is the complex acoustic velocity and equals the plate surface velocity. The acoustic intensity is then integrated over the plate surface to calculate the radiated sound power. The radiated sound power for the incident diffuse field is calculated by summing up that of each individual plane wave, using Eq. ( 7 ), then divided by the number of waves based on the diffuse field model. As with the modal model, 100 realizations of the simulation were considered and the results were averaged to approximate a diffuse field. Figure 11 (b) shows the calculated result at the first resonant frequency of the stiffened plate for one realization of the diffuse field. 


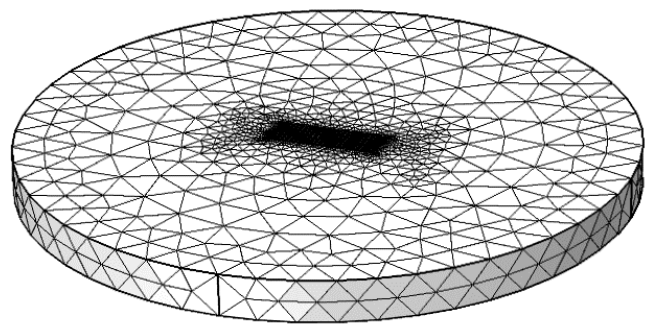

(a)

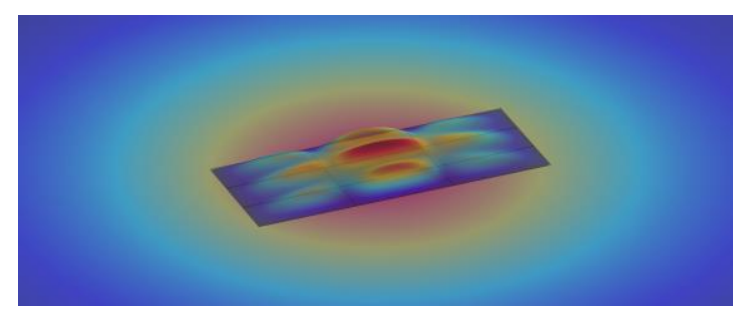

(b)

Figure 11 A coupled FE-BE numerical model for the baffled stiffened plate subjected to diffuse field. (a) The meshed model.

(b) The displacement response for the incident diffuse field of amplitude $94 \mathrm{~dB}$ at the first resonance frequency of the stiffened plate $(62.5 \mathrm{~Hz})$.

Figure 12 (a) and (b) show the comparison of the results for the reference point displacement and the TL to the diffuse field between the proposed modal model and the coupled FE-BE model. Both the vibroacoustic coupling and the modal cross terms are included in these two models.

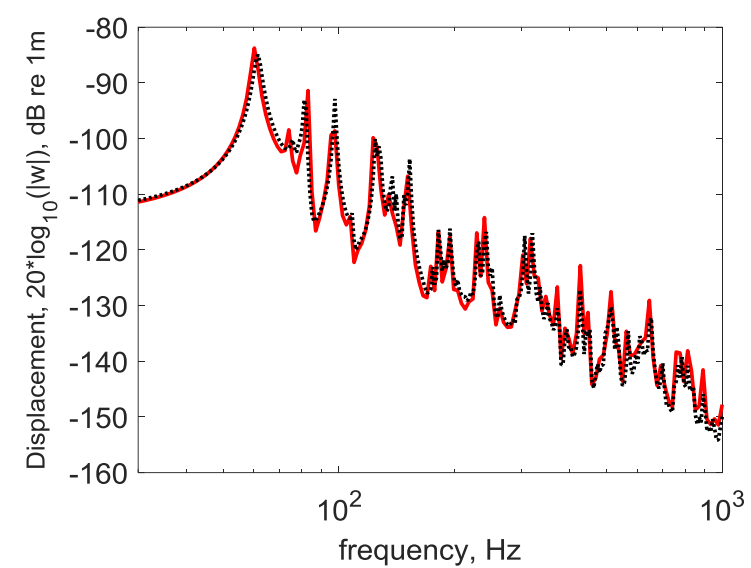

(a)

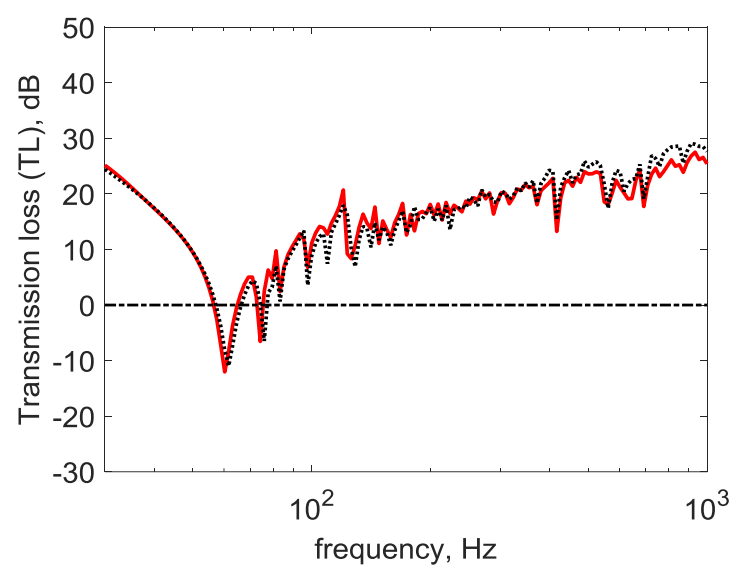

(b)

Figure 12. The comparison of the results for the stiffened plate displacement response (a) and the TL (b) for the diffuse field between the proposed modal model (in black, dotted lines) and the coupled FE-BE model (in red, solid lines). Horizontal dash-dot line: $0 \mathrm{~dB}$ line. 
A good agreement for both the displacement response and the TL from the two models is found over the entire frequency range presented in Figure 12. It can therefore be concluded that the model proposed here can accurately predict the vibroacoustic behaviour of flat plates excited by different forms of acoustic field.

The computation time of the proposed model and the FE-BE model were compared in the dual 2.0 GHz Intel Xeon E5-2670 processors with 40 cores and 394 GB memory. The CPU time of approximately 1190 hours was required for the FE-BE simulation, while only 2 hours was used for the proposed model, which was 590 times faster than the fully coupled FE-BE model. The solution time can be further reduced if the modal cross term contributions are neglected. In this study, the computational time for the FE-BE model has been reduced to 164 hours by utilizing the parallelized simulation in an Iridis High Performance Computing (HPC).

\section{Conclusions}

This paper presents a linear modal model for prediction of the fully coupled vibroacoustic response of stiffened plates subject to incident plane and diffuse sound fields. A relationship between the JAF and the modal radiation efficiency was derived, which simplifies the analysis and reduces the computational cost. The numerical results give a systematic explanation of the factors influencing the displacement response and the TL for a baffled, orthogonally stiffened plate, including the form of the acoustic excitation, vibroacoustic coupling and modal cross terms. The results show that the stiffeners can enhance vibroacoustic coupling leading to a significant reduction of the dominant resonance level, which is about $11 \mathrm{~dB}$ in the presented model. Different acoustic excitation types and the modal cross terms affect more the sound transmission loss than the vibration response. Although the presence of stiffeners in general will reduce the overall vibration response levels, both the effects of different excitation types and the effects of modal cross term contribution are preserved for either the unstiffened or stiffened plates. A comparison was also made between the proposed models and a 
coupled FE-BE model. Overall, good agreement was achieved with a significant reduction in the computational cost.

\section{Acknowledgement}

Dong Zhao acknowledges the project "Acoustic fatigue prediction for advanced aerospace structures", funded by the BISE-FEE Joint Laboratory, for its financial support of the research. The authors acknowledge that L. Dozio provided us with the analytical model of stiffened plates [22]. The authors would like to acknowledge the use of the IRIDIS HPC Facility and associated support services at the University of Southampton.

\section{References}

[1] G. Rodrigues and J. Santiago-Prowald, "Qualification of Spacecraft Equipment: RandomVibration Response Based on Impedance/Mobility Techniques," Journal of Spacecraft and Rockets, vol. 45, no. 1, pp. 104-115, 2008, doi: 10.2514/1.29734.

[2] H. Djojodihardjo, "BEM-FEM acoustic-structural coupling for spacecraft structure incorporating treatment of irregular frequencies," International Astronautical Federation 58th International Astronautical Congress 2007, vol. 8, 2007.

[3] C. Mei and Y. Shi, "Modeling of Structural-Acoustic Interaction Using Coupled FE/BE Method and Control of Interior Acoustic Pressure Using Piezoelectric Actuators," NASA Langley Research Center, United States, NASA-CR-204986, 1997.

[4] F. J. Fahy and P. Gardonio, Sound and structural vibration : radiation, transmission and response. Oxford, UK: Academic Press, 2007.

[5] A. Sestieri and A. Carcaterra, "Vibroacoustic: The challenges of a mission impossible?," Mechanical Systems and Signal Processing, vol. 34, no. 1, pp. 1-18, 2013, doi: https://doi.org/10.1016/j.ymssp.2012.08.010.

[6] B. Rafaely, "Spatial-temporal correlation of a diffuse sound field," The Journal of the Acoustical Society of America, vol. 107, no. 6, pp. 3254-3258, 2000, doi: 10.1121/1.429397.

[7] D. J. Thompson, "Noise control," in Fundamentals of sound and vibration. London: CRC Press, 2015, ch. 5, p. 292.

[8] R. W. Gordon and J. J. Hollkamp, "Reduced order models for acoustic response prediction," Air Force Research Libratory, United States, AFRL-RB-WP-TR-2011-3040, 2011.

[9] J. J. Hollkamp, "Experiences with nonlinear modeling and acoustic fatigue," Journal of Sound and Vibration, Article vol. 437, pp. 437-446, 2018, doi: 10.1016/j.jsv.2018.04.029.

[10] A. Powell, "On the fatigue failure of structures due to vibrations excited by random pressure fields," The Journal of the Acoustical Society of America, vol. 30, no. 12, pp. 1130-1135, 1958, doi: 10.1121/1.1909481.

[11] E. J. Richards and D. J. Mead, "Elements of periodic vibration theory," in Noise and acoustic fatigue in aeronautics. London: Wiley, 1968, ch. 13, pp. 296-297.

[12] C. E. Wallace, "Radiation Resistance of a Rectangular Panel," The Journal of the Acoustical Society of America, vol. 51, no. 3B, pp. 946-952, 1972, doi: 10.1121/1.1912943. 
[13] C. E. Wallace, "Structural response and acoustic fatigue for random progressive waves and diffuse fields," Journal of Spacecraft and Rockets, vol. 22, no. 3, pp. 340-344, 1985, doi: 10.2514/3.25753.

[14] R. D. Blevins, "An approximate method for sonic fatigue analysis of plates and shells," (in English), Journal of Sound and Vibration, vol. 129, no. 1, pp. 51-71, 1989.

[15] J. P. Coyette, G. Lielens, M. Robbe, and P. Neple, "An efficient method for evaluating diffuse field joint acceptance functions for cylindrical and truncated conical geometries," Journal of the Acoustical Society of America, vol. 117, no. 3, pp. 1009-1019, 2005, doi: 10.1121/1.1850368.

[16] A. Mejdi and N. Atalla, "Dynamic and acoustic response of bidirectionally stiffened plates with eccentric stiffeners subject to airborne and structure-borne excitations," Journal of Sound and Vibration, vol. 329, no. 21, pp. 4422-4439, 2010, doi: https://doi.org/10.1016/i.jsv.2010.04.007.

[17] L. Meirovitch, Analytical methods in vibrations. New York: Macmillan, 1967.

[18] J. W. S. B. Rayleigh, The Theory of Sound. Macmillan, 1896.

[19] D. J. Thompson and P. Nelson, "Fundamentals of Acoustics," in Fundamentals of sound and vibration. London: CRC Press, 2015, ch. 2, p. 63.

[20] L. Dozio and M. Ricciardi, "Free vibration analysis of ribbed plates by a combined analyticalnumerical method," Joumal of Sound and Vibration, vol. 319, no. 1-2, pp. 681-697, 2009, doi: 10.1016/j.jsv.2008.06.024.

[21] T. Beberniss and R. Gordon, "Finite Element Prediction of Acoustic Radiation Damping," presented at the 49th AIAA/ASME/ASCE/AHS/ASC Structures, Structural Dynamics, and Materials Conference, Schaumburg, USA, 2008.

[22] Acoustics module user's guide, version 5.3a. (2017). COMSOL Inc. [Online]. Available: www.comsol.com

[23] D. J. Ewins, "Applications," in Modal testing: theory and practice. UK: Research Studies Press, 1984, ch. 6, p. 225.

[24] D. Zhao, G. Squicciarini, and N. S. Ferguson, "The acoustic response of stiffened plates," Journal of Physics: Conference Series, vol. 1264, p. 012041, 2019, doi: 10.1088/17426596/1264/1/012041.

[25] D. J. Thompson, P. Gardonio, and J. Rohlfing, "Can a transmission coefficient be greater than unity?," Applied Acoustics, vol. 70, no. 5, pp. 681-688, 2009. [Online]. Available: https://eprints.soton.ac.uk/65271/.

[26] E. C. Sewell, "Transmission of reverberant sound through a single-leaf partition surrounded by an infinite rigid baffle," Journal of Sound and Vibration, vol. 12, no. 1, pp. 21-32, 1970, doi: https://doi.org/10.1016/0022-460X(70)90046-5.

[27] D. Zhao, N. S. Ferguson, and G. Squicciarini, "Acoustic response of thin-walled, orthogonally stiffened cylinders," IOP Conference Series: Materials Science and Engineering, vol. 657, p. 012007, 2019, doi: 10.1088/1757-899x/657/1/012007. 